the latter kind, the companion is generally bluish, and the number of blue companions increases rapidly with the distance, so that the close pairs are generally of the same, more or less white colour, and not very different in magnitude, whereas the wide pairs are of complementary colours, and the companion much fainter than the primary. We see then that the brightness is, on the whole, more different, the more different the colour is-a circumstance first pointed out by Struve. Now it is well known that the colour of a solid or liquid cooling body passes from white through yellow to red, and these are the colours in which the single stars, without exception, appear to us, whereas the blue and purple stars are found only as small companions to brighter stars. Holden, in Washington, has suggested that these colours are due to absorptive atmospheres-an opinion, the decision of which we leave to spectroscopists. Pickering, in Cambridge, U.S., has shown, from the absence of polarisation, that they do not shine with reflected light.

The last remarks remind us of the planets, that, no doubt, revolve round those distant suns, and derive from them their light and heat. When we reflect upon the complicated nature of the orbits, which the planets and comets describe round our sun, we get some idea of how remarkable must be the nature of those curves that planets describe round double suns, compared to which the motion of our moon is simple, and easily understood. Perhaps the same comets that disappear to our gaze, leaving the sphere of attraction of the sun, are attracted, and for a time become members of those wonderful systems. Our mathematics do not suffice for solving the problems that are thus suggested, but it is not unlikely that, unless situated very close to one or other of the suns-so close that the other appears not much larger than a star, though comparable in brightness to the nearest sun -the planets are whirled out in the cold space by the gravitational influence of the other sun, as very likely many a minor planet was ejected from the solar system under the influence of Jupiter. What must be the nature of those worlds illuminated by two different suns, one yellow and another purple? Now rises the one, and all is clothed in yellow, now the other, and illuminated from complementary sources, every object appears in its natural colour. Then sets the yellow sun, and what must be the diversity of the effects as it approaches the horizon! And behold nature puts on a purple mantle. Then also that sun sets, and in the darkness of night, though there is seldom night where there are two suns, the starry heavens are seen much the same there as here, except perhaps for moons reflecting light from the differently-coloured suns. But stars that seem large to us are hardly visible there, while our sun is perceived in the telescopes of the mysterious beings that inhabit those strange globes as only a faint star, and metaphysicians there prove from a priori considerations to their attentive pupils, that no life could bask in the feeble glare of a single sun-how all would famish but for the opposite effects of the two suns. And no doubt! life there is heightened, and the wonders of nature are unravelled by aid of and under the influence of the energy of several suns, more highly developed science, seeing how glorious is the creation on this poor orb, that is kept alive by but one. Not only the play of colour must there be more varied than here, but phenomena of which we have not the faintest idea, must be produced also through the action of heat, electricity, and magnetism. ${ }^{1}$ Indeed, upon subjects like these, science gives no information, and we may therefore give our fancy free reins. Oh! that we did possess the power of appreciating these things like the divine Milton, when in truly prophetic strain he wrote :-

$$
\begin{aligned}
& \text { "Other suns, perhaps, } \\
& \text { With their attendant moon", thou wilt descry, }
\end{aligned}
$$

\footnotetext{
The tides upon the planets caused by the two suns must be very interest-
}

Communicating male and female light,

Which two great sexes animate the world,

Stored in each orb, perhaps, with some that live ;

For such vast room in nature unpossess'd,

By living soul, desert and desolate,

Only to shine, yet scarce to contribute

Each orb a glimpse of light, conveyed so far

Down to this habitable, which returns

Light back to them, is obvious to dispute."

\section{W. DOBERCK}

\section{ON THE PHOTOGRAPHIC SPECTRUM OF COMET (WELLS) $I_{.,} 1882^{1}$}

O

$\mathrm{N}$ May $3 \mathrm{I}$ I obtained a photograph of the spectrum of this comet, with an exposure of one hour and a quarter. On the same plate I took a spectrum of $a$ Ursæ majoris for comparison. The comet's spectrum on the plate consists of a strong continuous spectrum extending from about $F$ to a little beyond $H$. I am not able to distinguish any of the Fraunhofer lines in this continuous spectrum. The slit was rather more open than was the case in photographing the spectrum of the comet of last year; this would make these lines less distinct, but the lines $G$ and $H$ are well seen in the star's spectrum taken under the same conditions. We may therefore conclude that the part of the comet's original light which gives a continuous spectrum is much stronger relatively to the reflected solar light in this comet, than was the case in the comet of last year, and for this reason the Fraunhofer lines are not distinguishable.

Observations of the visible spectrum had already shown that the comet differs remarkably from the hydrocarbon type common to all the comets, some twenty, which have appeared since spectrum analysis has been applied to these bodies.

The photographic spectrum shows, as was to be expected, that this essential difference of spectrum exists also in the more refrangible region. The very strong ultra-violet group assigned to cyanogen is not to be seen on the plate, and the bright groups between $\mathrm{G}$ and $h$, and between $h$ and $\mathrm{H}$ do not appear to be present.

The head of the comet was in sharp focus upon the slit, and the continuous spectrum with defined edges corresponds to the nucleus which in this comet was very distinct. In this continuous spectrum at least five separate places of greater brightness are seen, which very probably represent groups of bright lines, though they are not sufficiently distinct in the photograph to admit of resolution. That this interpretation is correct, seems probable, from the circumstance that these groups, as shown in the diagram, project beyond the strong continuous spectrum on one side. This side corresponds to where the light of the coma, on the side of the nucleus next the sun, falls upon the slit. We learn, therefore, that the light of this part of the coma consists for the most part in this part of the spectrum of these groups, as here on the plate only an exceedingly faint continuous spectrum can be seen.

It is not possible to measure with any useful accuracy the beginnings and endings of the groups, as they are too faint at these points. Measures as accurate as the circumstances would permit have been taken of the brightest parts of the groups. The wave-lengths of these brightest $\lambda 4253$ $\lambda 4412$

parts are : $\lambda 4507$

$\lambda 4634$ $\lambda 4769$

In the visible spectrum the bright lines of sodium

ing. Of course they also produce tides upon each other, and their spots present no doubt most peculiar features. It is not unlikely that in some cases the phenomena presented by new as well as by some variable stars are to be explained as the effect of tides caused by darker companions.

I Substance of note read befire the Royal Society, June 15,1882 . 
appear to have been strong, and it may be that some of ago that the spectra of the periodic meteors are different the light of some of the groups may be due to this for different swarms, and it does not seem surprising that substance. we have now a comet, the matter of the nucleus of which

Prof. A. Herschel and Dr. von Konkoly showed long under the sun's heat shows an essential chemical differ-

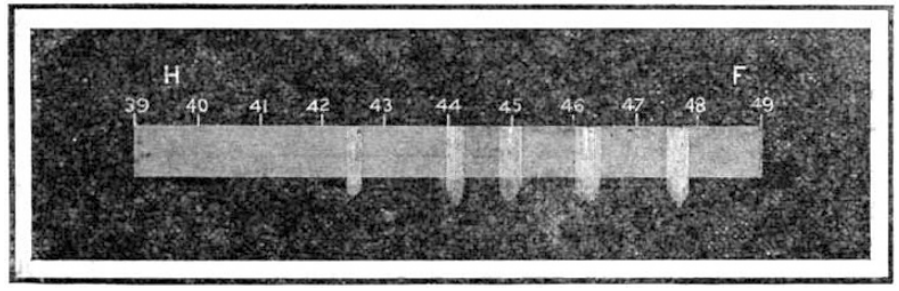

ence from the long series of hydrocarbon comets which have appeared since $186_{4}$.

taken. The comet was then $42,380,000$ miles distant from the sun, while the comet of last year was $69,420,000$ miles

Mr. Hind has kindly furnished me with the distance of when I obtained the photograph of its spectrum.

this comet from the sun at the time the photograph was

WILLIAM HUGGINS

$K A B A T H ' S$ ELECTRTC ACCUMULATORS

$A^{T}$ intervals since the introduction of accumulators or A secondary batteries by $M$. Gaston PJanté, various modifications have been made with a view to constructing cells on a commercial scale. The most renowned of these modifications was that of Faure, who applied red lead to the surface of the lead plates, so as to furnish a greater thickness of the spongy mass that is the effective agent in the storage process. In this development he had been anticipated by d'Arsonval, who sought to increase the efficacy of the oxidised lead electrode by covering it with a layer of lead dross. De Meritens, Tommasi, and others have worked in another direction by employing many sheets of lead foil presenting a large amount of surface, whilst Swan, Sillon, and Volckmar bave taken another departure in applying perforated plates of lead with disintegrated material packed into the interstices. From our contemporary La Nature we have borrowed the accompanying illustration of another form of accumulator due to M. Nicolas de Kabath, whose suggestion is

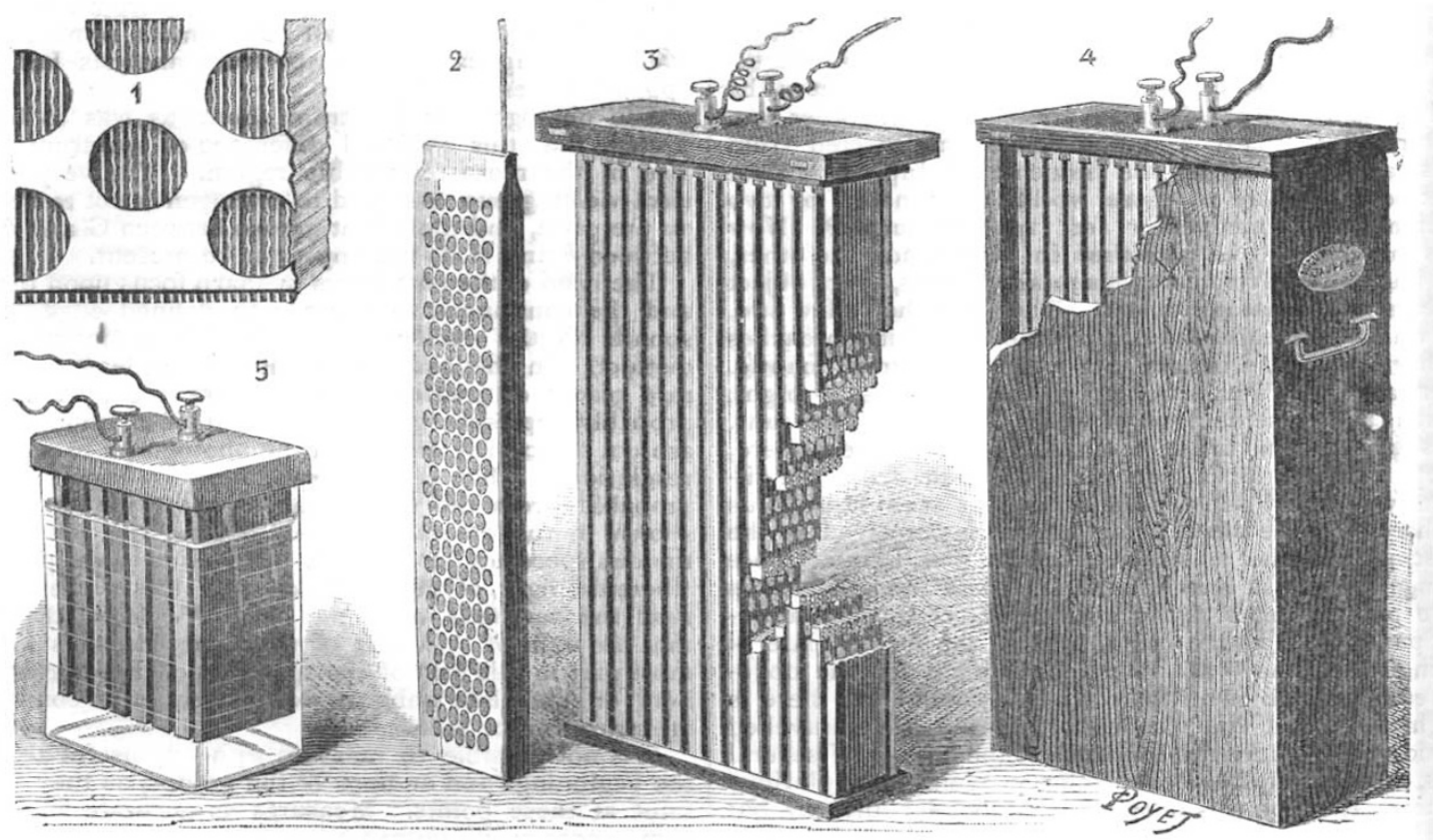

Kabath's Accumulators made of currugated lead plates. Figs. r, 2, 3 showing details of manufacturz. Fig. 4, Commercial Accumulator. Fig. 5, Laboratory Accumulator.

to employ thin plates of gauffered or corrugated lead, so as to secure a large amount of effective surface. The thin corrugated sheets are cut into narrow strips and packed between two stouter sheets of lead pierced with holes, through which the dilute acid liquor can circulate freely. The details of construction will be amply explained by the figures. The object of the perforated exterior is to prevent the cells from becoming short circuited by the possible falling down of the thin corrugated strips which are rapidly disintegrated during the preliminary charging or "formation" of the cell. The perforated leaden cases are themselves placed side by side in an appropriate cell, and are connected so that they serve alternately as positive and negative plates. Smaller cells are used for laboratory work. No detail's have yet been published, so far as we are aware, of their performances or capability of retaining the charge that has been imparted. 